\title{
Do-not-resuscitate orders as part of advance care planning in patients with COPD
}

\author{
Jo Raskin ${ }^{1,3}$, Kristina Vermeersch ${ }^{1,2,3}$, Stephanie Everaerts ${ }^{1,2}$, \\ Pascal Van Bleyenbergh ${ }^{1,2}$ and Wim Janssens ${ }^{1,2}$
}

Affiliations: ${ }^{1}$ University Hospitals Leuven, Dept of Respiratory Diseases, Leuven, Belgium. ${ }^{2} \mathrm{KU}$ Leuven, Laboratory of Respiratory Diseases, Dept of Chronic Diseases, Metabolism and Ageing, Leuven, Belgium. ${ }^{3}$ These authors contributed equally.

Correspondence: Wim Janssens, University Hospitals Leuven, Dept of Respiratory Diseases, KU Leuven, Herestraat 49, 0\&NI, Box 706, B-3000 Leuven, Belgium. E-mail: wim.janssensquzleuven.be

ABSTRACT There is growing awareness of the need for advance care planning in patients with chronic obstructive pulmonary disease (COPD). However, do-not-resuscitate (DNR) order implementation remains a challenge in clinical practice.

We retrospectively analysed an observational cohort of 569 COPD patients with 2.5-8 years of follow-up in secondary care, to evaluate potential determinants and the prognostic significance of DNR order implementation and specification.

345 patients $(61 \%)$ had no DNR order, of whom $27 \%$ died during a median (interquartile range (IQR)) follow-up of 1935 (1290-2448) days. 194 (39\%) patients had a DNR order, of whom 17 had the order at baseline and 82\% died (median (IQR) follow-up 528 (137-901) days), while 177 received an order during follow-up and 76\% died (median (IQR) follow-up 1322 (721-2018) days). 88\% of DNR orders were implemented during hospitalisation. $58 \%$ of the patients with a DNR order died within the first year after admission; of them, 66\% died in the hospital. Age, forced expiratory volume in $1 \mathrm{~s}$, chronic oxygen dependency and previous mechanical ventilation were significantly and independently associated with DNR order implementation. DNR order specification was significantly associated with increased mortality, even after adjustment for age and disease severity.

These findings identify DNR orders as independent determinants of mortality, mainly implemented just before death.

@ERSpublications

DNR orders in COPD patients are preferentially given to older patients with severe disease and are usually implemented during hospitalisation, close to the moment of death. Early end-of-life care planning in COPD remains difficult and challenging http://ow.ly/BGJk30hJCZ5

Cite this article as: Raskin J, Vermeersch K, Everaerts S, et al. Do-not-resuscitate orders as part of advance care planning in patients with COPD. ERJ Open Res 2018; 4: 00116-2017 [https://doi.org/ 10.1183/23120541.00116-2017].

Received: Sept 202017 | Accepted after revision: Dec 162017

W. Janssens reports receiving grants and personal fees for consultancy from Boehringer Ingelheim, AstraZeneca, Novartis, GlaxoSmithKline and Chiesi, outside the submitted work.

Support statement: This work was supported by the KU Leuven Astra Zeneca Chair in Respiratory Pathophysiology. AstraZeneca was not involved in the study design, in the collection, analysis, and interpretation of data, in the writing of the manuscript, or in the decision to submit the manuscript for publication. Funding information for this article has been deposited with the Crossref Funder Registry.

Copyright $\odot$ ERS 2018. This article is open access and distributed under the terms of the Creative Commons Attribution Non-Commercial Licence 4.0. 


\section{Introduction}

Chronic obstructive pulmonary disease (COPD) is a progressive and incurable lung disease, with cigarette smoking as its most important causative factor [1]. It is characterised by chronic airflow limitation and gradual destruction of normal lung tissue, which is associated with increasing symptom load, emotional distress and social isolation. For many patients, maximal treatment only leads to modest and incomplete symptom relief, leaving them with significantly reduced quality of life [2].

The disease trajectory is often unpredictable, as its slow functional decline can be complicated by sudden and potentially life-threatening exacerbations [3], resulting in accelerated disease progression and unexpected mortality. When compared to cancer, patients with end-stage COPD tend to experience morbidity for a longer period of time. Moreover, they are more likely to be hospitalised and transferred to the intensive care unit (ICU) and are less likely to realise that death is imminent $[4,5]$. Nonetheless, suffering from a nonmalignant disease, they receive considerably less palliative and end-of-life (EOL) care [4].

Fortunately, awareness of the need for advance care planning is growing. However, much of our experience is derived from the needs of patients with cancer, and its applicability is often questioned. The importance of open discussions between patients and healthcare professionals as the EOL approaches is heavily emphasised [6]. An essential feature is discussing do-not-resuscitate (DNR) order preferences, such as the desire to undergo low- or high-burden interventions to sustain life. It is critical to discuss these preferences proactively and to have a DNR order implemented before the patient deteriorates to a critical level, which in COPD is not straightforward [7]. The clinical course of cancer is often associated with a first period of progressive dependency on caretakers, followed by EOL care, whereas in COPD, patients' care needs may fluctuate repeatedly from minor to major for several years as health deteriorates with exacerbations, but may improve with rehabilitation and medical or surgical intervention. As a result, COPD becomes as much as a way of life as an illness. Exacerbations may not be experienced as advancing disease, but rather as temporary setbacks, attributed to specific activities, weather changes, environmental factors, faltering self-management or infection. Likewise, recovery from an exacerbation may be misinterpreted as a sign of cure, obscuring the overall decline in health. The sense of what is normal, and the tolerability of health problems and interventions both evolve with the disease [7].

The unpredictable disease trajectory and the uncertainty of prognosis, as well as the patient's potentially poor understanding of COPD as a life-limiting disease challenge the appropriate and timely implementation of DNR orders [3,4]. COPD progresses at a highly variable rate, and the outcomes of life-threatening exacerbations are currently determined by last-minute, rushed decisions regarding life support with little information about patients' wishes [8]. The present study aimed to evaluate the time point and potential determinants of DNR order implementation, as well as the impact of DNR order specification on the prognosis in COPD patients.

\section{Methods}

\section{Study population and design}

The study population was derived from an existing observational cohort (Leuven COPD cohort), comprising patients with a COPD diagnosis at enrolment based on a smoking history of $\geqslant 15$ pack-years, age $>50$ years and a post-bronchodilator forced expiratory volume in $1 \mathrm{~s}$ (FEV1)/forced vital capacity ratio $<0.7$. The study is prospectively investigating clinical, molecular and genetic traits in COPD and lung cancer, and is approved by the UZ-KU Leuven Ethics Committee (ML11081; September 30, 2007). All patients provided informed consent.

Between October 1, 2007 and September 9, 2013, 600 COPD patients without lung cancer at enrolment were prospectively enrolled at the outpatient clinic of the University Hospital Leuven (Leuven, Belgium), a tertiary-care hospital fully equipped for respiratory care. To obtain clinical follow-up data of $\geqslant 2.5$ years, all patients enrolled before April 1, 2013 were included in the present retrospective cross-sectional study $(n=569)$. All patients were routinely followed at the outpatient clinic at 0.5 - or 1-yearly intervals, interrupted by hospitalisations.

\section{Data collection}

Data were collected retrospectively by reviewing the electronic medical records. The patient's DNR order (the specification (table 1), reason and location where it was implemented) was recorded, along with demographic and disease severity characteristics at enrolment. In the electronic records, a DNR module enabled physicians to specify the DNR order by indicating which life-sustaining interventions (e.g. cardiopulmonary resuscitation, intubation and mechanical ventilation) were no longer feasible. The module did not allow noninvasive ventilation (NIV) to be specified separately; however, common practice was to specify NIV preferences in the text box used to comment on the reason for DNR order implementation. This text box permitted the documentation of more than one reason and its completion 


\begin{tabular}{|c|c|c|}
\hline & Details & Patients \\
\hline $\begin{array}{l}\text { DNR 0: do not restrict } \\
\text { therapy }\end{array}$ & $\begin{array}{l}\text { Explicit statement not to withhold any life-sustaining } \\
\text { interventions }\end{array}$ & $22(11)$ \\
\hline DNR 1: do not resuscitate & Further specified as: & \\
\hline DNR 1a & No CPR & $47(24)$ \\
\hline DNR 1b & No CPR + no intubation + NIV to be considered & $77(40)$ \\
\hline $\begin{array}{l}\text { DNR 2: do not extend } \\
\text { therapy }\end{array}$ & No CPR + no intubation + no NIV & $46(24)$ \\
\hline DNR 3: discontinue therapy & $\begin{array}{c}\text { No CPR + no intubation + no NIV + withdrawal of current } \\
\text { treatment }{ }^{\#}\end{array}$ & $2(1)$ \\
\hline
\end{tabular}

was not mandatory. Mortality was considered as outcome measure; the date, location and cause of death were recorded for patients who died in the hospital. For patients who died elsewhere, only the date was retrieved from the electronic records, since the location and cause of death marked on the official mortality register in Belgium could not be accessed. The database was completed retrospectively, with data on DNR orders and mortality obtained until October 1, 2015.

\section{Statistical analysis}

Statistical analyses were performed using SPSS (version 20; IBM, Armonk, NY, USA). Two-sided p-values $<0.05$ were considered statistically significant.

Parametric data, assessed by Shapiro-Wilk normality test, were compared using one-way ANOVA or Fisher's exact test. p-values were estimated via a Monte Carlo simulation with 5000 replicates. Nonparametric data were analysed by Kruskal-Wallis statistics. A multivariate logistic regression model was fitted, in which all variables were included. Kaplan-Meier curves and Cox regression assessed the influence of DNR order specification (due to small sample size of DNR $3(n=2)$, DNR 2 and 3 were combined) on survival in patients with and without a DNR order. The study period ran from the time of DNR order implementation or enrolment (whichever came first), until death or October 1, 2015. Pairwise log rank comparisons and a Bonferroni correction were conducted, with statistical significance accepted at $\mathrm{p}<0.01$. Furthermore, the frequency distribution for mortality was calculated in patients with and without a DNR order on a nonlinear time scale. The following time points were assessed: prior to, within $48 \mathrm{~h}$ after and at days 3-10, 11-30 (1 month), 31-90 (3 months), 91-365 (1 year), 366-730 (2 years) and $>2$ years after the date of DNR order implementation or enrolment. The frequency distributions were compared using Mann-Whitney-Wilcoxon statistics. The risk periods for both groups were expressed graphically as a smoothed curve fitted to the frequency distributions. In addition, cumulative risk was assessed, the distributions of which were compared using the two-sample Kolmogorov-Smirnov test.

\section{Results}

\section{Patient characteristics}

24 patients were excluded for undergoing lung transplantation, therefore no longer having COPD, and six patients were considered lost to follow-up as their survival status could not be determined. Table 2 summarises the characteristics of the 539 patients used for analysis.

$36 \%(n=194)$ of the patients had a DNR order, of which $9 \%(n=17)$ had the order at baseline, i.e. the moment of enrolment in the observational cohort. At the moment of database completion, an additional 177 patients had received a first DNR order, two of whom had already died at home when the order was implemented ( 5 and 12 days after death) during revision of the hospital discharge letter. During the follow-up period, modifications were made to $71 \mathrm{DNR}$ orders; once to explicitly confirm the current DNR order, in two cases to withdraw a previous DNR order and to further limit interventions in the remaining 68 cases.

Patients with a DNR order had a median (interquartile range (IQR)) age of 76.8 (70.9-82.8) years (versus 72.6 (66.0-79.2) years, $\mathrm{p}<0.0001$ ), a median (IQR) FEV1 of 41 (32-53)\% predicted (versus 55 (41-67)\% pred, $\mathrm{p}<0.0001)$ and a median (IQR) diffusing capacity of the lung for carbon monoxide of $43(32-53) \%$ pred (versus $53(40-67) \%$ pred, $\mathrm{p}<0.0001)$. They had higher median (IQR) Medical Research Council 


\section{TABLE 2 Patient characteristics}

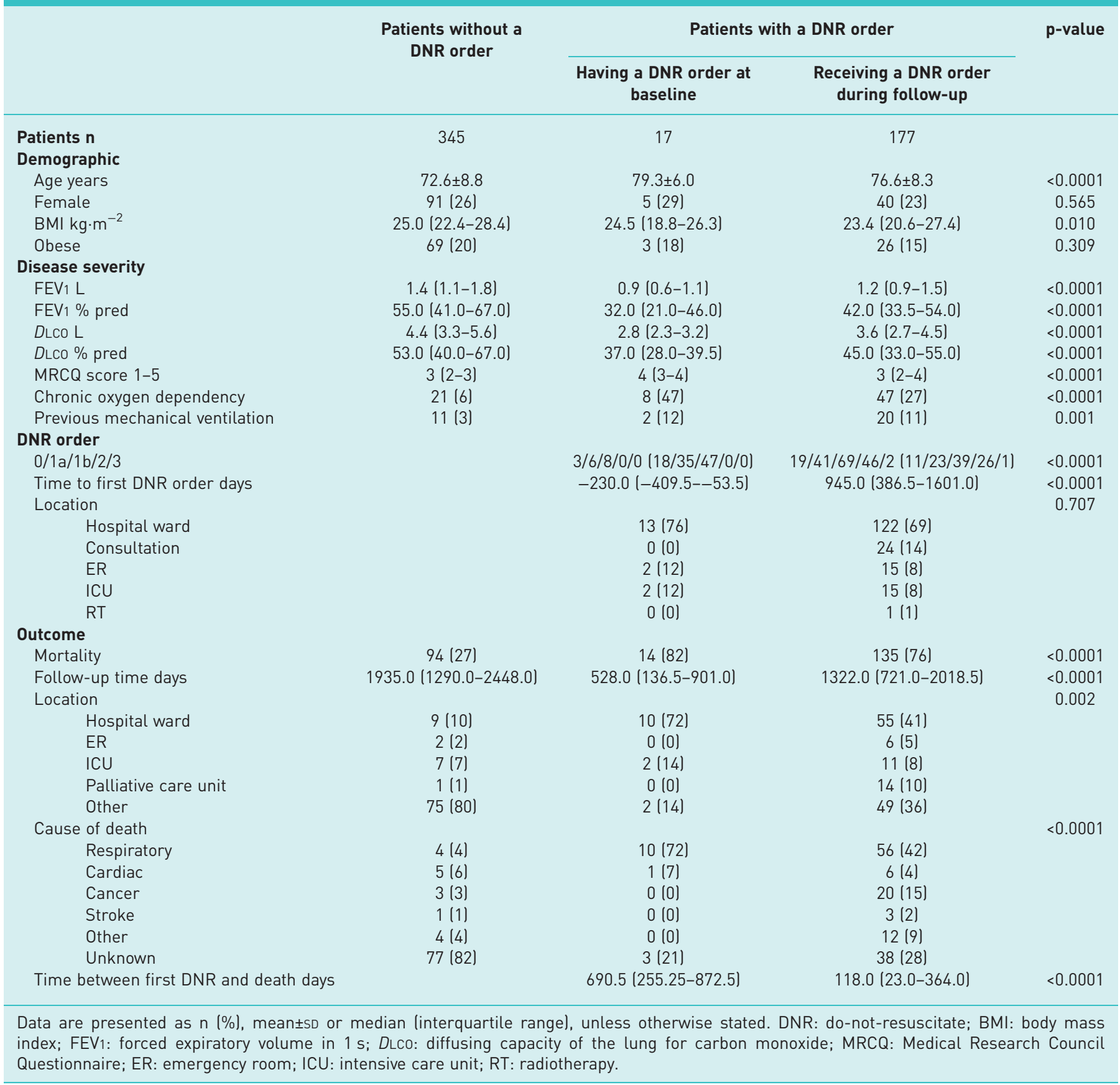

Questionnaire (MRCQ) scores (3 (2-4) versus $3(2-3), \mathrm{p}<0.0001)$, were more likely to be chronically oxygen dependent $(28 \%$ versus $6 \%, \mathrm{p}<0.0001)$ and had been more often previously mechanically ventilated ( $11 \%$ versus $3 \%, \mathrm{p}<0.0001)$. In the subgroup of patients with a DNR order at baseline, these characteristics were even more pronounced.

$40 \%$ of the patients with a DNR order had a DNR $1 \mathrm{~b}(\mathrm{n}=77)$, with the remaining majority having a less restrictive DNR 1a $(n=47,24 \%)$ or a more restrictive DNR $2(n=46,24 \%)$. A DNR 0 was recorded in $11 \%$ $(n=22)$, while only $1 \%(n=2)$ had a DNR 3. In the subgroup of patients who had a DNR order at baseline, almost $50 \%$ had a DNR $1 \mathrm{~b}(\mathrm{n}=8)$, with the remainder having a less restrictive DNR $1 \mathrm{a}(\mathrm{n}=6,35 \%)$ or DNR $0(n=3,18 \%)$.

Approximately $70 \%$ of the DNR orders were implemented on the hospital ward $(n=135), 12 \%$ during a consultation visit $(n=24), 9 \%$ in the emergency room (ER) $(n=17)$ or in the ICU $(n=17)$ and $1 \%$ during 
TABLE 3 Reason for implementing a first do-not-resuscitate (DNR) order ${ }^{\#}$

Patients

Poor respiratory status

126

Patient preference

Noncurable malignancy

Cardiac disease

Poor functional status

Advanced age

Cognitive decline

Persistent smoking

Request for euthanasia

Noncompliance with treatment

Obesity

Cachexia

Declined for lung transplantation

Traumatic previous intubation

Other medical condition

Unknown

Data are presented as $\mathrm{n} . \mathrm{N}=194 .{ }^{*}$ : the reason for implementing a first DNR order was recorded in a text box which permitted the documentation of more than one reason, and its completion was not mandatory.

radiotherapy for cancer $(n=1)$. There was no significant difference between the two subgroups. The median time to first DNR order during follow-up was 945 days (95\% CI 386.5-1601.0 days). Poor respiratory status, patient preference and noncurable malignancy as a sole reason or as one of several were recorded as the top three reasons for implementing a first DNR order, applicable in $65 \%(n=126), 19 \%$ $(n=37)$ and $13 \%(n=25)$ of the patients, respectively. In $14 \%(n=28)$, no reason was recorded. Table 3 provides the full list of reasons and contributing factors.

\section{Determinants of DNR order implementation}

A multivariate logistic regression model was fitted to identify independent determinants associated with the implementation of DNR orders (table 4). There was no effect of demographic variables, apart from a $6 \%$ increase in odds of DNR order implementation for every year increase in age. Chronic oxygen dependency and previous mechanical ventilation were associated with a three-fold increased odds, while every percentage increase in $\mathrm{FEV} 1$ was associated with a $2 \%$ decrease in odds.

\section{Mortality}

On October 1, 2015, 243 patients had died, of whom 61\% had a DNR order ( $n=149$; eight had a DNR 0 and 141 had limiting life-sustaining interventions). In patients with a DNR order at baseline and those receiving it during follow-up, mortality was significantly higher $(82 \%$ and $76 \%$ versus $27 \%, \mathrm{p}<0.0001)$, and the median follow-up time significantly shorter (528.0 days (95\% CI 136.5-901.0) and 1322.0 days (95\% CI 721.0-2018.5) versus 1935.0 days (95\% CI 1290.0-2448.0); $\mathrm{p}<0.0001$ ) when compared to patients

TABLE 4 Multivariate logistic regression model: determinants associated with

do-not-resuscitate (DNR) order implementation

\begin{tabular}{|c|c|c|}
\hline & Odds ratio $(95 \% \mathrm{Cl})$ & p-value \\
\hline Age years & $1.063(1.037-1.090)$ & $<0.001$ \\
\hline Male versus female & $1.043(0.638-1.705)$ & 0.866 \\
\hline BMI $\mathrm{kg} \cdot \mathrm{m}^{-2}$ & $0.982(0.938-1.027)$ & 0.417 \\
\hline FEV $1 \%$ pred & $0.979(0.966-0.992)$ & 0.002 \\
\hline Dıco \% pred & $0.988(0.972-1.003)$ & 0.127 \\
\hline MRCQ score 3-5 versus $1-2$ & $1.389(0.883-2.185)$ & 0.155 \\
\hline Chronic oxygen dependency? Yes versus no & $3.641(1.995-6.646)$ & $<0.001$ \\
\hline Previous mechanical ventilation? Yes versus no & $3.398(1.469-7.860)$ & 0.004 \\
\hline
\end{tabular}

Bold type represents statistical significance. BMI: body mass index; FEV1: forced expiratory volume in $1 \mathrm{~s}$; $D L C O$ : diffusing capacity of the lung for carbon monoxide; MRCQ: Medical Research Council Questionnaire. 
without a DNR order. Location and cause of death were significantly different, with $81 \%$ of the patients without a DNR order dying outside the hospital; consequently, cause of death was not known primarily. In patients with a DNR order, death mainly occurred on the hospital ward $(n=65,44 \%)$ or in the ICU $(n=13,9 \%)$. The leading cause of death was respiratory related. In the subgroup of patients with a DNR order at baseline, these characteristics were even more pronounced. Notably, in the patients receiving a DNR order during follow-up, cancer as a second cause of death and mortality in the palliative care unit accounted for $15 \%$ and $10 \%$ of deaths, respectively. The median (IQR) time between first DNR order and death was 163.0 (26.0-420.5) days, which was significantly shorter in patients receiving a DNR order during follow-up (118.0 (23.0-364.0) days) compared to patients with a DNR order at baseline (690.5 (255.3-872.5) days).

\section{Efficacy implementation of DNR order}

In addition to the time between first DNR order and death, the correlation between the location of DNR order implementation and location of death was further investigated to provide some insight into the efficacy of DNR orders.

Out of the 20 patients who died in the ICU, 13 (65\%) had a DNR order, of whom one was implemented in the ER just before ICU admission to withhold cardiopulmonary resuscitation (CPR) and intubation and five were implemented in the ICU in response to the patient's deteriorating condition. The remaining seven DNR orders were implemented on the hospital ward. The seven patients without a DNR all died on mechanical ventilation, although only two were admitted for respiratory reasons (acute exacerbation of COPD or pneumonia).

An additional 12 patients who received a DNR order in the ICU survived their ICU stay, of whom five subsequently died on the hospital ward. Seven patients were successfully discharged home; four of them were treated in the ICU with mechanical ventilation or NIV later in the disease course.

Of the eight patients that died in the ER, five (62.5\%) had received a DNR order $\geqslant 1$ month previously in the context of advance care planning.

An additional 15 patients who received a DNR order in the ER survived their ER stay. 10 of them were hospitalised on the respiratory ward and were successfully discharged home (two were readmitted rapidly, one died $<2$ months later in a palliative care unit and one died at home within 1 month).

\section{Survival}

The 17 patients with a DNR order at baseline, as well as the two patients having a DNR order implemented after date of death, were excluded from the survival analyses. The Kaplan-Meier survival plot is shown in figure 1. Patients without a DNR order had a mean survival of 2366.7 (95\% CI 2269.0-2464.3) days, whereas patients with a DNR 0, 1a, $1 \mathrm{~b}$ and $2 / 3$ had a mean survival of 685.0 (95\% CI 462.8-907.2) days, 1065.0 (95\% CI 681.4-1448.6) days, 381.7 (95\% CI 251.9-511.6) days and 343.1 (95\% CI 192.3-493.8) days, respectively. All pairwise comparisons revealed a significant difference, apart from the survival distributions for DNR 0 versus DNR $1 \mathrm{a}(\mathrm{p}=0.470)$ and DNR $1 \mathrm{~b}$ versus $\mathrm{DNR} 2 / 3(\mathrm{p}=0.361)$ (data not shown). The Cox regression analysis showed that DNR 0, 1a, 1b and 2/3 compared to no DNR order were significantly associated with higher mortality risk after adjustment for age, FEV1, MRCQ score, chronic oxygen dependency and previous need for mechanical ventilation (table 5).

\section{Risk period}

The frequency distribution for mortality on a nonlinear time scale was calculated for patients with and without a DNR order (table 6), and was plotted to provide a graphical representation of the risk period for each population (figure 2). The 17 patients with a DNR order at baseline were excluded from the analyses.

In patients with a DNR order, the frequency distribution was shifted significantly toward earlier time points with a median survival time of 182.0 (95\% CI 34.0-510.5) days (versus 1935.0 (95\% CI 1290.0-2448.0); $\mathrm{p}<0.0001)$. The observed mortality per time point was found to increase up to the first year after DNR order implementation, culminating to $58 \%$ mortality 1 year after DNR order implementation. At the moment of database completion, $76 \%$ of the patients with a DNR order had died (versus $27 \%, \mathrm{p}<0.0001$ ). At any time point, the observed mortality was higher in patients with a DNR order, apart from the 1.9-fold higher mortality in patients without a DNR order 2 years after enrolment. Significant differences were observed for cumulative mortality.

\section{Discussion}

In this study, COPD patients receiving a DNR order were characterised as older patients with more severe lung disease. The main reason for DNR order implementation was "poor respiratory status" and the most 


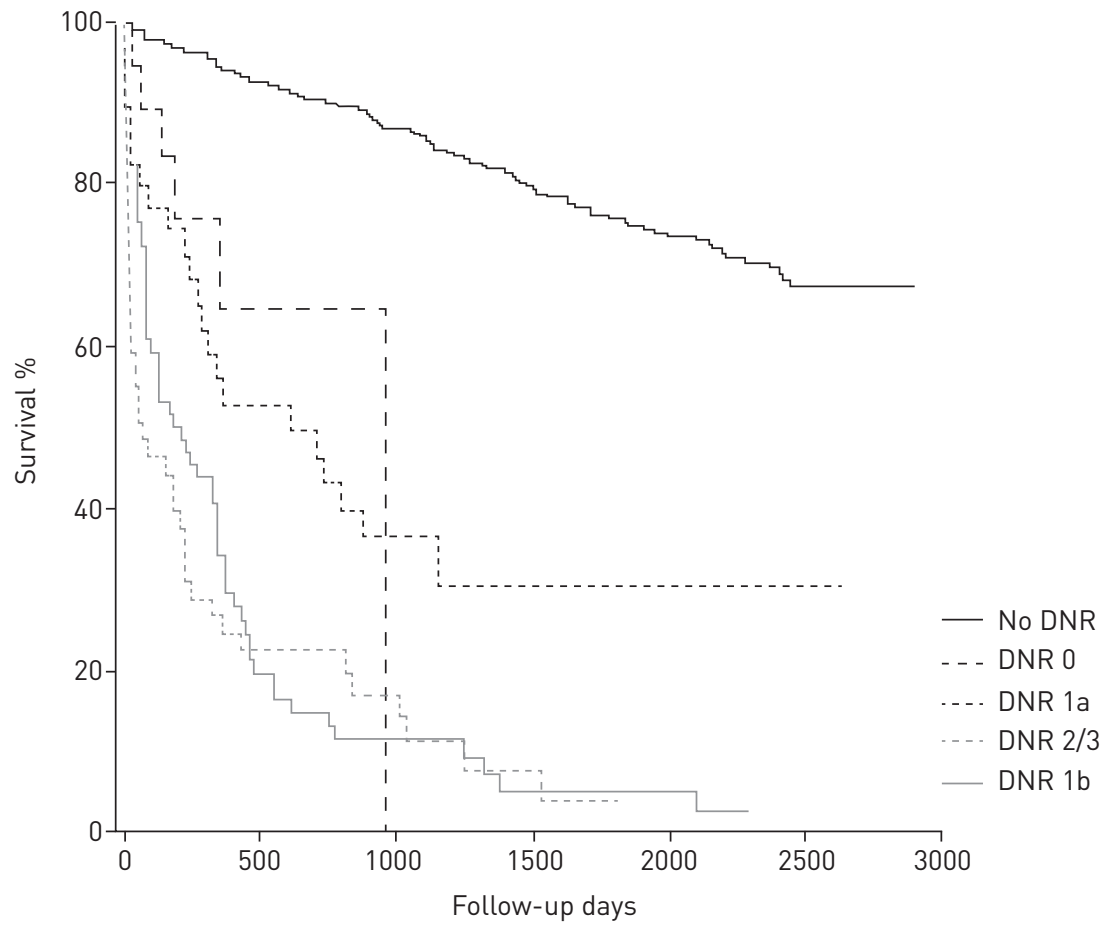

$\begin{array}{lccccccc}\text { Subjects at risk } \mathrm{n} & & & & & & \\ \text { No DNR } & 345 & 319 & 292 & 223 & 159 & 78 & 0 \\ \text { DNR 0 } & 19 & 5 & 0 & 0 & 0 & 0 & 0 \\ \text { DNR 1a } & 40 & 16 & 7 & 3 & 2 & 1 & 0 \\ \text { DNR 1b } & 69 & 12 & 5 & 2 & 1 & 0 & 0 \\ \text { DNR 2/3 } & 47 & 10 & 5 & 1 & 0 & 0 & 0\end{array}$

FIGURE 1 Influence of do-not-resuscitate (DNR) order specification on overall survival in chronic obstructive pulmonary disease (COPD) patients (log rank test $\mathrm{p}<0.001$ ). Pairwise log rank comparisons revealed significantly different survival distributions at a Bonferroni corrected $p<0.01$ level, with exception for DNR 0 versus DNR 1a $(p=0.470)$ and DNR $1 b$ versus DNR $2 / 3(p=0.361)$. Due to small sample size of DNR $3(n=2)$, DNR 2 and 3 were combined.

frequent DNR order was "no CPR nor intubation, NIV to be considered". While perhaps not surprising, these findings are a reflection of the life-limiting disease that is COPD, in which acute or chronic respiratory failure is often seen as the terminal phase of the disease [9]. It is frequently associated with comorbidities, the most serious and prevalent being cardiovascular disease and malignancy [10], as we

\section{TABLE 5 Cox regression analysis of do-not-resuscitate (DNR) order implementation ${ }^{\#}$}

\begin{tabular}{lcc} 
& Hazard ratio (95\% CI) & p-value \\
\hline No DNR & Ref. & \\
DNR 0 & $4.984(2.121-11.71)$ & $<\mathbf{0 0 0 1}$ \\
DNR 1a & $4.217(2.597-6.845)$ & $<\mathbf{0 0 0 1}$ \\
DNR 1b & $8.699(5.860-42.91)$ & $<\mathbf{0 0 0 1}$ \\
DNR 2/3 & $10.36(6.808-15.79)$ & $<\mathbf{0 . 0 0 1}$ \\
Age years & $1.030(1.013-1.047)$ & $\mathbf{0 . 0 0 1}$ \\
FEV1\% pred & $0.986(0.978-0.995)$ & $\mathbf{0 . 0 0 2}$ \\
MRCQ score 3-5 versus 1-2 & $1.494(1.095-2.038)$ & $\mathbf{0 . 0 1 1}$ \\
Chronic oxygen dependency? Yes versus no & $1.024(0.714-1.467)$ & 0.899 \\
Previous mechanical ventilation? Yes versus no & $1.101(0.676-1.792)$ & 0.699 \\
\hline Bold type represents statistical significance. \# : adjusted for age, forced expiratory volume in $1 \mathrm{~s} \mathrm{(FEV1),}$ \\
Medical Research Council Questionnaire (MRCQ) score, chronic respiratory dependency and previous need \\
for mechanical ventilation. ref.: reference against which other DNR values are compared.
\end{tabular}




\begin{tabular}{|c|c|c|c|}
\hline & $\begin{array}{l}\text { Patients without a } \\
\text { DNR order }\end{array}$ & $\begin{array}{l}\text { Patients with a } \\
\text { DNR order }\end{array}$ & p-value \\
\hline Patients n & 345 & 177 & \\
\hline Survival time days & $1935.0(1290.0-2448.0)$ & $182.0(34.0-510.5)$ & $<0.0001$ \\
\hline Mortality per time point & & & $<0.0001$ \\
\hline Prior to & $0(0)$ & $2(1.1)$ & \\
\hline Within $48 \mathrm{~h}$ after & $0(0)$ & 7 (3.9) & \\
\hline Between 3 and 10 days after & $1(0.3)$ & 15 (8.5) & \\
\hline Between 11 and 30 days ( 1 month) after & $1(0.3)$ & $18(10.2)$ & \\
\hline Between 31 and 90 days ( 3 months) after & $5(1.5)$ & 23 (12.9) & \\
\hline Between 91 and 365 days ( 1 year) after & $13(3.8)$ & 37 (20.9) & \\
\hline Between 366 and 730 days ( 2 years) after & $12(3.5)$ & $16(9.0)$ & \\
\hline After 730 days until database completion & $62(17.9)$ & $17(9.6)$ & \\
\hline Still alive on the date of database completion & $251(72.8)$ & $42(23.7)$ & \\
\hline Cumulative mortality ${ }^{\#}$ & & & $<0.0001$ \\
\hline Prior to & $0(0)$ & $2(1.1)$ & \\
\hline Within $48 \mathrm{~h}$ after & $0(0)$ & $9(5.1)$ & \\
\hline Within 10 days after & $1(0.3)$ & $24(13.6)$ & \\
\hline Within 30 days ( 1 month) after & $2(0.6)$ & $42(23.7)$ & \\
\hline Within 90 days ( 3 months) after & $7(2.0)$ & $65(36.7)$ & \\
\hline Within 365 days ( 1 year) after & $20(5.8)$ & $102(57.6)$ & \\
\hline Within 730 days ( 2 years) after & 32 (9.3) & $118(66.7)$ & \\
\hline After 730 days until database completion & $94(27.2)$ & 135 (76.3) & \\
\hline Still alive on the date of database completion & $251(72.8)$ & $42(23.7)$ & \\
\hline
\end{tabular}

found reflected in the full list of reasons for DNR order implementation. The presence of comorbidities is the strongest predictor of in-hospital mortality and 6-month survival after hospitalisation for an exacerbation [11]. While NIV to treat respiratory failure has been shown to decrease the length of hospitalisation and improve survival, it is well known that intubation may lead to a poorer prognosis [12]. Advance care planning should therefore incorporate a multidisciplinary approach that takes into consideration all of the components of the disease [9].

$70 \%$ of the DNR orders were implemented during hospitalisation, compared to only $12 \%$ under stable conditions at ambulatory follow-up. Generally, recommendations advise against making EOL decisions
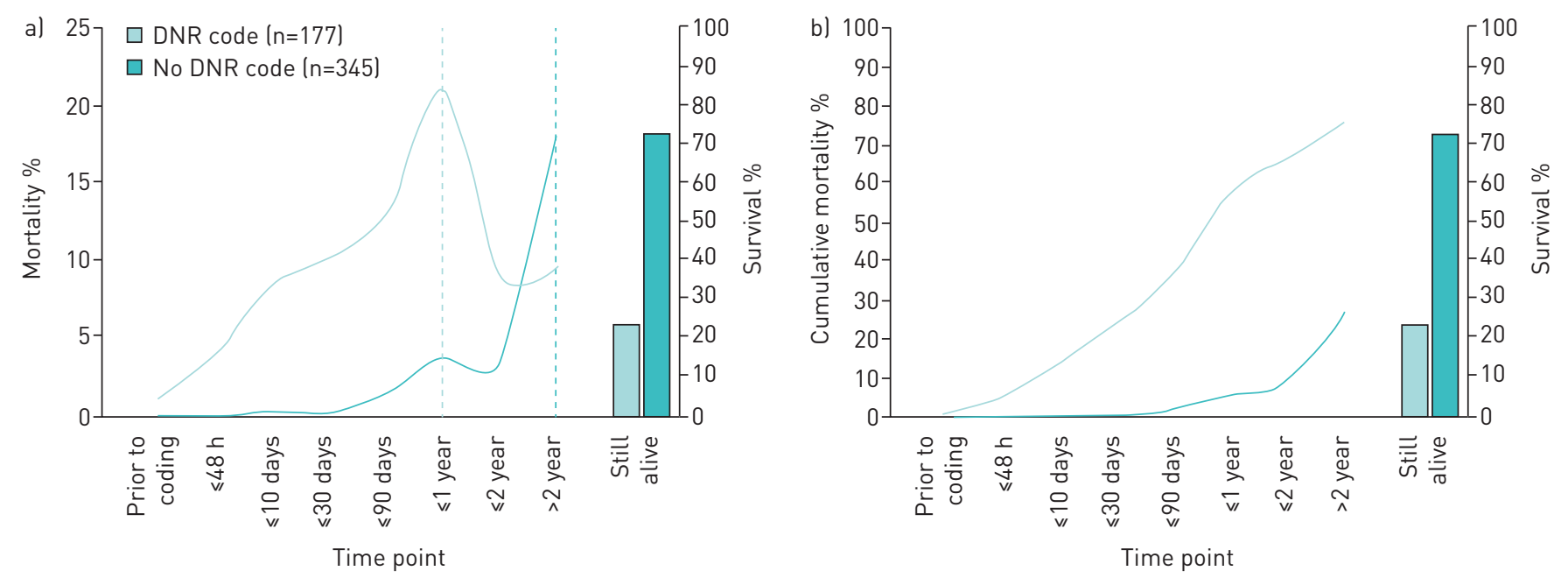

FIGURE 2 a) Mortality per time point $(p<0.0001)$; b) cumulative mortality $(p<0.0001)$ on a nonlinear time scale according to do-not-resuscitate (DNR) status in patients with chronic obstructive pulmonary disease. The median is indicated by the dotted line. 
during hospitalisation as physical and mental dysfunction may prohibit thoughtful decision-making [13]. Ideally, DNR order preferences should be previously discussed in an outpatient setting, and are to be reviewed and updated upon hospital admission. Its relevance in COPD is reinforced by a recent European audit on exacerbations requiring hospitalisation, revealing their association with $12 \%$ mortality and $35 \%$ risk of readmission within 3 months after discharge [14]. The discrepancy in timing between our practice and the current recommendations reflects the difficulty of timely and appropriate DNR order implementation in a chronic terminal disease challenged by unpredictable disease trajectory, uncertainty of prognosis and different patients' perspectives. The most commonly reported barriers for EOL care communication by physicians are lack of time, anxiety about taking away hope and the assumption that the patient is not ready to talk about EOL care [15], whereas patients would rather concentrate on staying alive than talk about death, and have a fear of abandonment by their regular physician at EOL [2, 16]. There is no doubt that all of these reasons contributed to the limited number of EOL discussions during our ambulatory follow-up, and which can certainly be improved. Nevertheless, hospitalisation appears to provide the desired time and setting for both patients and physicians to overcome some of the barriers to discussion of DNR order preferences. Necessity or urgency during hospital admission probably drives both patients and healthcare professionals to EOL decisions. Once implemented, reassessments are performed quite regularly. $37 \%$ of DNR orders were modified during follow-up, mainly to adapt them to declining health and functional status. Overall, failure to discuss life-saving interventions or therapeutic restrictions should be avoided, as it results in more patient suffering and therapeutic obstinance [17]. An American Thoracic Society statement endorses offering palliative care to all respiratory disease patients in every stage of disease, based on the needs and preferences of the patients and their families [18]. Advance directives have been shown to be fairly consistent over time, implicating that well-informed decisions about treatment preferences, even early in the course of disease, are valuable [18].

Patients receiving a DNR order have a high probability of dying, as evidenced by a $77 \%$ mortality and a median time-to-death of 163 days after first DNR order implementation. Death occurred mainly in the hospital, and the leading cause was respiratory related. Prognostication in COPD is challenging, due to the unpredictable disease trajectory and highly variable rate of progression. The observation that $27 \%$ of patients without a DNR order died during follow-up may reflect these difficulties. In the SUPPORT trial, $14 \%$ of patients who were likely to survive for 2 months ( $75 \%$ probability) had a written DNR order, compared to $67 \%$ of patients who were unlikely (25\% probability) to survive that long. Despite the different setting, these data corroborate our findings that DNR orders are mainly implemented in patient groups with the highest risk of mortality. While prognostic tools, such as the BODE (body mass index, airflow obstruction, dyspnoea, exercise capacity) index, are superior to FEV1 decline alone in predicting all-cause and respiratory-related mortality, they are not developed nor validated for short-term mortality during and immediately after hospital admission [19]. In a multivariate model, we identified age, FEV1, chronic oxygen dependency and previous need for mechanical ventilation as the main determinants for DNR order implementation, which all have been validated as prognostic factors for mortality. Moreover, a more restrictive DNR order was found to be associated with increasing mortality risk, independent of age and disease severity. Pairwise comparisons of the DNR order specifications further highlighted the worse outcome of COPD patients for whom intubation was no longer considered feasible. These data support literature findings suggesting that DNR orders are independent determinants of short-term mortality [20]. In fact, they incorporate multiple estimates on patient characteristics (disease severity, comorbidity, age,...) and physician's clinical experience, but also patient preferences. Furthermore, once appointed, restrictive DNR orders directly impact survival, as some of the lifesaving interventions are declined.

Despite correcting for variables commonly associated with increased risk of mortality, we identified an independent association between DNR order implementation and increased risk of mortality. The exact nature of this association is ambiguous given the strong correlation between the factors: a DNR order will ultimately be implemented due to the impending death of a patient, and likewise, the restrictions in life-sustaining interventions implemented by a DNR order are likely to accelerate death. This is reflected by our data: in the ICU, implementation of a DNR order usually occurred in response to the patient's deteriorating condition, whereas in the ER it was more in line with advance care planning. On the hospital ward and before transfer to the ICU, DNR orders were implemented anticipating a poor outcome, consequently leading to a greater likelihood of mortality in the ICU.

Our study has several limitations. Due to its retrospective nature, data on patient and physician barriers to EOL discussions, as well as readmissions, are lacking. We only addressed patient records of the hospital and were not able to collect information on EOL conversations nor on EOL decisions that were taken in primary care. Finally, as the study is based on a large but single centre experience, our findings cannot be generalised to all other care settings with potentially different organisations and cultures. 


\section{Conclusion}

While guidelines advise engagement in advance care planning in the early stages of a disease, we conclude that DNR orders in COPD patients are preferentially given to older patients with more severe disease and are usually implemented during hospitalisation (e.g. exacerbations), close to the moment of death. These observations indicate that early EOL care planning in a COPD cohort remains difficult and challenging.

\section{Acknowledgements}

KV and SE are supported as doctoral candidates by the Flemish Government Agency for Innovation by Science and Technology and the Fund for Scientific Research Flanders, respectively. WJ is supported as a postdoctoral clinical researcher by the Fund for Scientific Research Flanders. KV and JR contributed equally towards data analysis and writing of the paper. JR performed the data collection. PVB and SE contributed substantially to data interpretation. WJ takes responsibility for the content of the manuscript, provided the study idea and critically reviewed the manuscript.

The data have been presented in part at the European Respiratory Society congress (London, 2016).

\section{References}

1 Laniado-Laborin R. Smoking and chronic obstructive pulmonary disease (COPD). Parallel epidemics of the 21st century. Int J Environ Res Public Health 2009; 6: 209-224.

2 Curtis JR. Palliative and end-of-life care for patients with severe COPD. Eur Respir J 2008; 32: 796-803.

3 Patel K, Janssen DJA, Curtis JR. Advance care planning in COPD. Respirology 2012; 17: 72-78.

4 Gardiner C, Gott M, Payne S, et al. Exploring the care needs of patients with advanced COPD: an overview of the literature. Respir Med 2010; 104: 159-165.

5 Spathis A, Booth S. End of life care in chronic obstructive pulmonary disease: in search of a good death. Int $J$ Chron Obstruct Pulmon Dis 2008; 3: 11-29.

6 Momen N, Hadfield P, Kuhn I, et al. Discussing an uncertain future: end-of-life care conversations in chronic obstructive pulmonary disease. A systematic literature review and narrative synthesis. Thorax 2012; 67: 777-780.

7 Giacomini M, DeJean D, Simeonov D, et al. Experiences of living and dying with COPD: a systematic review and synthesis of the qualitative empirical literature. Ont Health Technol Assess Ser 2012; 12: 1-47.

8 Gore JM, Brophy CJ, Greenstone MA. How well do we care for patients with end stage chronic obstructive pulmonary disease (COPD)? A comparison of palliative care and quality of life in COPD and lung cancer. Thorax 2000; 55: 1000-1006.

9 Carlucci A, Guerrieri A, Nava S. Palliative care in COPD patients: is it only an end-of-life issue? Eur Respir Rev 2012; 21: 347-354.

10 Decramer M, Janssens W. Chronic obstructive pulmonary disease and comorbidities. Lancet Respir Med 2013; 1 73-83.

11 Simonds AK. Ethics and decision making in end stage lung disease. Thorax 2003; 58: 272-277.

12 Conti G, Antonelli M, Navalesi P, et al. Noninvasive vs. conventional mechanical ventilation in patients with chronic obstructive pulmonary disease after failure of medical treatment in the ward: a randomized trial. Intensive Care Med 2002; 28: 1701-1707.

13 Fitten JL, Waite MS. Impact of medical hospitalization on treatment decision-making capacity in the elderly. Arch Intern Med 1990; 150: 1717-1721.

14 López-Campos JL, Hartl S, Pozo-Rodriguez F, et al. European COPD Audit: design, organisation of work and methodology. Eur Respir J 2013; 41: 270-276.

15 Houben CHM, Spruit MA, Wouters EFM, et al. A randomised controlled trial on the efficacy of advance care planning on the quality of end-of-life care and communication in patients with COPD: the research protocol. $B M$ J Open 2014; 4: e004465.

16 Knauft E, Nielsen EL, Engelberg RA, et al. Barriers and facilitators to end-of-life care communication for patients with COPD. Chest 2005; 127: 2188-2196.

17 Claessens MT, Lynn J, Zhong ZS, et al. Dying with lung cancer or chronic obstructive pulmonary disease: insights from SUPPORT. J Am Geriatr Soc 2000; 48: S146-S153.

18 Lanken PN, Terry PB, DeLisser HM, et al. An official American Thoracic Society clinical policy statement: palliative care for patients with respiratory diseases and critical illnesses. Am J Respir Crit Care Med 2008; 177 912-927.

19 Sin DD, Anthonisen NR, Soriano JB, et al. Mortality in COPD: role of comorbidities. Eur Respir J 2006; 28 $1245-1257$.

20 Hakim RB, Teno JM, Harrell FE Jr, et al. Factors associated with do-not-resuscitate orders: patients' preferences, prognoses, and physicians' judgments. Ann Intern Med 1996; 125: 284-293. 